\title{
URBAN LANDSCAPE SPATIAL PATTERN ESTIMATION OF CITIES IN SHANDONG PROVINCE USING NIGHTTIME LUMINOSITY DATA
}

\author{
Junfu Fan ${ }^{\text {a b, }}$, Huixin $\mathrm{He}^{\mathrm{a}}$, Taoying $\mathrm{Hu}^{\mathrm{a}}$, Guihua $\mathrm{Li}^{\text {a }}$, Huixian Gao ${ }^{\text {a }}$, Xiaowei Zhao ${ }^{\mathrm{c}}$ \\ ${ }^{a}$ School of Architectural Engineering, Shandong University of Technology, Zibo, 255049, China - fanjf@sdut.edu.cn; (hehx_sdut, \\ huty_sdut, ligh_sdut, gaohx_sdut)@163.com \\ ${ }^{\mathrm{b}}$ State Key Laboratory of Resources and Environmental Information System, Institute of Geographical Sciences and Natural \\ Resources Research, Chinese Academy of Sciences, Beijing, 100101, China \\ c College of Geoexploration Science and Technology, Jilin University, Changchun, 130026, China - zhaoxw16@mails.jlu.edu.cn
}

\section{Commission IV, WG IV/3}

KEY WORDS: DMSP/OLS, urbanization, landscape metrics, spatial patterns analysis, Shandong province

\begin{abstract}
:
China's cities have been undergoing rapid and intense urbanization processes in the past few decades. Shandong is a coastal province which is located in East China with big economy and population scales, and which also plays an important role in the rapid process of China's modernization. The DMSP/OLS dataset has been widely used for the urban development assessments in long time-series and large spatial scales situations. In this paper, we used a time series of nighttime light data to estimate the landscape spatial pattern changes of cities in Shandong province from 1994 to 2012. Nine landscape metrics were calculated and analyzed to figure out the spatial patterns of urban area developments of the cities in Shandong province. The landscape metrics include the number of patches (NP), the landscape total area (TA), the aggregation index (AI), the largest patch index (LPI), the mean patch area (AREA_MN), the landscape shape index (LSI), the total edge length (TE), the edge density (ED), and the mean radius of gyration (GYRATE_MN). The experimental results reveal that, in 1994-2012, the total urban area of cities in Shandong province expanded for 1.17 times, the average urban area increased by about $93.00 \%$, the average annual growth rate of the TE metric is $2.67 \%$, while the ED metric decreased about $1.44 \%$ annually. Bigger cities in this area show relative slower urbanization development processes, such as Jinan and Qingdao. Coastal cities represented much more rapid expansion velocities than inland cities. In the middle area of Shandong province, the connectivity between developed urban areas was constantly increased.
\end{abstract}

\section{INTRODUCTION}

In the last several decades, with the rapid growth of China's economy and continuous investments of infrastructure constructions, China's cities have been experiencing an intense developing process with remarkable urban built-up area expansions, energy consumptions and population migrations (Ma et al. 2012b). Shandong is a coastal province of China, and is a part of the East China region. After a period of political instability and economic hardship that began in the late 19th century, Shandong has emerged as one of the most populous (97.89 million inhabitants in 2014, about 55\% lives in urban areas) and most affluent provinces in China with a GDP of $¥ 5.942$ trillion in 2014 (National Bureau of Statistics of China 2016), or $\$ 967$ billion, making it become China's third wealthiest province. There are 17 prefecture-level cities in Shandong which have been experienced rapid developments driven by the basic state strategy of reform and opening-up in the last 38 years, especially in the recent 20 years. However, the spatial distribution of urban developments is varied between cities in this area (Yang et al. 2015). Furthermore, many social and environmental problems have been presented during the rapid urbanization processes in this area, such as soil pollution and degradation, excessive speed for rural to urban population migration, nature resource wastes (Ma et al. 2012a; Xiong et al. 2012; Deng et al. 2015). It is important to solve the "urban diseases" for the sustainable development of the province. The establishment of reasonable macro-control polices for sustainable development requires wide and deep understanding of many social economy aspects about this region. Consequently, it is necessary to evaluate the spatial and temporal patterns of cities in Shandong province to support policy making in environmental management and urban planning, in response to the remarkably rapid urbanization processes occurring in this region.

Remotely sensed nighttime light time series dataset derived from the Defense Meteorological Satellite Program's Operational Linescan System (DMSP/OLS) provides a straightforward way to analyze the relationship between urbanization and anthropogenic activities and has been extensively used in urban studies (Ma et al. 2012b; Fan et al. 2014). The DMSP/OLS sensor could detect low-level visible and near-infrared bands radiance signals at night and the composed stable nighttime light imageries have been widely used for urban development mapping (Elvidge et al. 1997; Small et al. 2005; Zhang et al. 2011; Zhou et al. 2014, Xu et al. 2014), city built-up area expansion trends exploring (Sutton 2003; Ma et al. 2016) and socioeconomic activities detecting (Doll et al. 2006; Elvidge et al. 2009). The nighttime light time series data also used for estimation of regional socioeconomic development inequality (Zhou et al. 2015). Previous study suggested that there is no single valid brightness value used to split the urban regions and rural regions in a large scale because of the "blooming" effects (Small et al. 2005) and regional development imbalance (Zhou et al. 2015). However, the low cost and easy for acquisition characters of the DMSP/OLS

* Corresponding author: Junfu Fan, fanjf@sdut.edu.cn 
stable light data have made it became an important data source for urban studies. And many studies also reveal a common phenomenon that pixels with higher DN values always are relative to highly urbanized areas; pixels with lower DN values are located in rural areas. It can be inferred that at relatively small spatial scales and with a long time span, cities with different urbanization expansion characters could be classified and the spatial characters of urban expansion of different cities could be extracted from the DMSP/OLS nighttime luminosity data. Different spatial patterns correspond to different socioeconomic conditions and different solutions for the "urban disease". Therefore, the study of the changes in urbanization spatial patterns of Shandong's cities using DMSP/OLS data provided decision makers with potential references for the establishment of regional sustainable developing strategies and policies.

In this paper, we focused on nine landscape metrics to analyze the differences of spatial patterns between 17 prefecture-level cities in Shandong province. The landscape metrics, including the number of patches (NP), the landscape total area (TA), the aggregation index (AI), the largest patch index (LPI), the mean patch area (AREA_MN), the landscape shape index (LSI), the total edge length (TE), the edge density (ED), and the mean radius of gyration (GYRATE_MN) are calculated by the FRAGSTATS 4.2 (McGarigal et al. 2012) software. We conducted a series of pixel level relative corrections and statistics to analyze the urban area landscape changes of cities in Shandong from 1994 to 2012.

\section{STUDY AREA, DATA AND METHODS}

\subsection{Study Area}

Shandong is located in East China with a second-largest population scale and third-largest economy scale in all of China's provinces and municipalities in 2014 (National Bureau of Statistics of China 2016). Shandong has played a major role in Chinese history since the beginning of Chinese civilization along the lower reaches of the Yellow River, and it served as a pivotal cultural and religious site for Taoism, Chinese Buddhism, and Confucianism. Shandong's location at the intersection of ancient as well as modern north-south and eastwest trading routes has helped to establish it as an economic center (Wikipedia 2016). Shandong has experienced rapid urbanization process with other areas of China in recent decades. Shandong is a coastal province which extends out to the Bohai Sea and Yellow Sea as a peninsula. It also borders Henan, Hebei, Jiangsu and Anhui. The Jingjiu Railway (BeijingKowloon) and Jinghu Railway (Beijing-Shanghai) are both major arterial railways that pass through the western part of Shandong (Wikipedia 2016). With its unique geographical location, Shandong holds a crucial position in China's transport industry. Furthermore, Shandong as the largest agricultural exporter in China is abundant with products, such as cotton and wheat.

At the end of 2013, Shandong’s GDP had reached $¥ 5.5$ trillion and its urbanization rate had stood at $39.20 \%$ with a population of more than 90 million, which means it had taken a giant leap forward in the process of urbanization. The spatial locations of the 17 prefecture-level cities in Shandong province were presented in Figure 1.

\subsection{Data}

Depending on the operational line-scan system (OSL), the defense meteorological satellite program (DMSP) has provided a new data source to obtain urban expansion information. The OLS sensors can generate global daytime and nighttime coverage of the earth every 24 hours. The OLS sensors' primary mission was the observation of nighttime moonlit cloud cover for global meteorological forecasting for the U.S. Air Force (Silvana et al. 2001). A constellation of two satellites provides for global coverage four times a day by dawn, day, dusk, and night (Elvidge et al. 1999). Since 2000, the National Geophysical Data Center's (NGDC) has published several versions of the data. The version 4 DMSP/OLS nighttime lights time series (V4DNLTS) dataset has three types of data. Among the data, nighttime stable light (NSL) contains lights from cities, towns and other sites with persistent lighting (Elvidge et al. 1997; Amaral et al. 2005; Pandey et al. 2013). The composed stable nighttime light data consists of $30 \times 30$ arc-seconds (approximately 1000 meters at the equator) (Ma et al. 2016). The range of the nighttime stable light data's digital number (DN) is 0 to 63 .

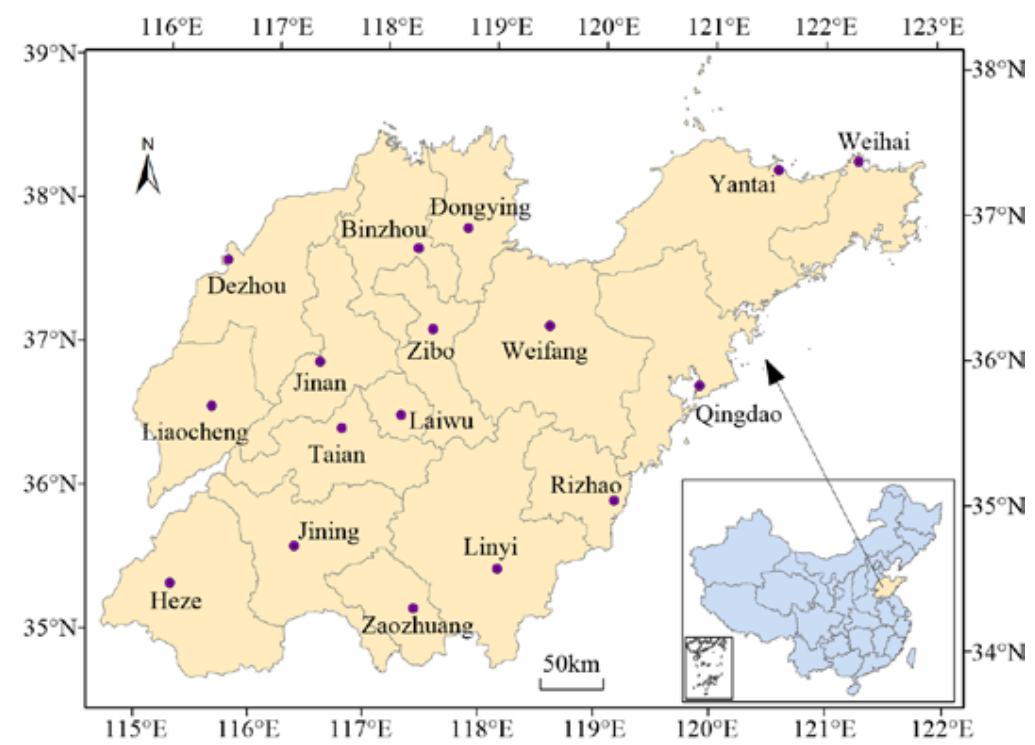

Figure 1. The location of Shandong province in China and the spatial distribution of its prefecture-level cities 
In this paper, in consideration of the continuity and comparability of data, we chose NSL data from 1994 to 2012 which was acquired by five DMSP satellites: F12(1994-1999), F14(2000-2003), F15(2004-2007), F16(2008-2009) and F18(2010-2012). The datasets can be obtained from the website of NGDC (http://www.ngdc.noaa.gov/dmsp/downloadV4compo sites.html).

\subsection{Methods}

The NSL data was widely used for rapid estimation of dynamics of global urban expansion at the spatial resolution of approximately $1 \mathrm{~km}$ (Zhang et al. 2011). For each year, NSL data acquired by different satellites has no strict intercalibration. Besides, OLS sensors have no on-board calibration. Those factors will produce unstable lit pixels in NSL data, which cause the lack of continuity and comparability. To improve the continuity and comparability of the NSL data sets, a series of essential systematic correction methods should be designed and developed. There were some crucial problems in the correction, such as the discrepancies between DN values of NSL data acquired from different satellites for the same year and the abnormal fluctuations of DN values acquired by the same satellites for different years (Liu et al. 2012). In this report, we employed a second-order model to implement the annual intercalibration of the nighttime light data with respect to the F121999 composite dataset (Elvidge et al. 2009). Based on the calibration results, a time series of luminosity data were assembled for subsequent analyses.

\section{URBAN AREA EXTRACTION}

\subsection{Intercalibration}

The NSL data set couldn't be used directly to map urban areas for the lack of continuity and comparability. Because DN values of NSL data were influenced by many factors, such as the sensors, the time when the satellite crossing. Even the same satellite in different year had discrepancies in DN values. Therefore, the long time series of NSL data which was obtained by different satellites should be calibrated. According to Elvidge's intercalibration model of global NSL data (Elvidge et al. 2009), the data of Sicily from satellite year F121999 showed the highest digital values and the most favorable characteristics. A second order regression model was proposed and applied for the calibration of each satellite year from 1994 to 2008 based on the reference of F121999 Sicily area data. Calibration parameters proposed by Elvidge (Elvidge et al. 2009) and Fan (Fan et al. 2013), as shown in Table 1, were used in this study for the inter-correction of the NSL time series data.

$$
D N_{a d j}=C_{0}+C_{1} \times D N+C_{2} \times D N^{2}
$$
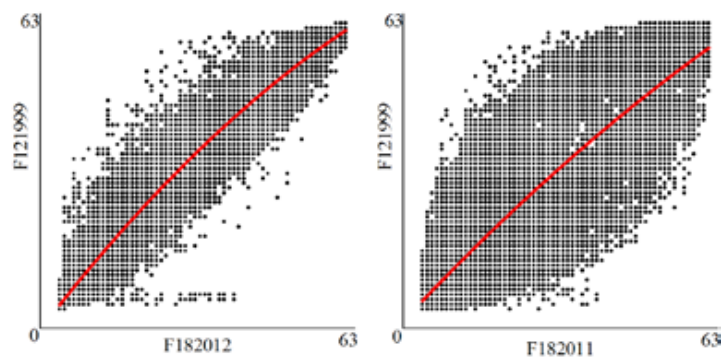

Figure 2. Regression results of F18(2011, 2012) and F121999
In addition to those coefficients from 1994 to 2010, calibration parameters of the year 2011 and 2012 were necessary. According to the model designed by Elvidge (Equation 1), we selected Sicily island as the calibration area and the F121994 image as the reference to perform an intercalibration experiment for F182011 and F182012 (Figure 2). The coefficients from 1994 to 2012, as listed in Table 1 and Table 2, were used to calibrate NSL data in the area of Shandong province.

\begin{tabular}{|c|c|c|c|c|}
\hline Satellite & Year & $C_{0}$ & $C_{1}$ & $C_{2}$ \\
\hline F12 & 1994 & 0.1651 & 1.1244 & -0.0018 \\
F12 & 1995 & 0.4103 & 1.2116 & -0.0035 \\
F12 & 1996 & 0.2228 & 1.2700 & -0.0040 \\
F12 & 1997 & -0.0008 & 1.1651 & -0.0023 \\
F12 & 1998 & 0.1535 & 1.0451 & -0.0009 \\
F12 & 1999 & 0 & 1 & 0 \\
F14 & 2000 & 0.1061 & 1.3877 & -0.0059 \\
F14 & 2001 & -0.2595 & 1.3467 & -0.0053 \\
F14 & 2002 & 0.4486 & 1.1983 & -0.0035 \\
F14 & 2003 & -0.2768 & 1.2838 & -0.0044 \\
F15 & 2004 & -0.3657 & 1.3772 & -0.0056 \\
F15 & 2005 & -0.6201 & 1.3504 & -0.0049 \\
F15 & 2006 & -0.6005 & 1.3551 & -0.0049 \\
F15 & 2007 & -0.1615 & 1.3960 & -0.0054 \\
F16 & 2008 & -0.1203 & 1.0155 & -0.0001 \\
F16 & 2009 & 0.9878 & 1.0610 & -0.0014 \\
F18 & 2010 & 2.5479 & 0.4911 & 0.0068 \\
\hline
\end{tabular}

Table 1. The coefficients from 1994 to 2010 derived by comparing data of Sicily with F121999

\begin{tabular}{|c|c|c|c|c|c|}
\hline Year & $C_{0}$ & $C_{1}$ & $C_{2}$ & $R^{2}$ & Number \\
\hline 2011 & 3.4701 & 0.6553 & 0.0030 & 0.7655 & 35174 \\
2012 & 2.0050 & 0.6110 & 0.0053 & 0.9377 & 36542 \\
\hline
\end{tabular}

Table 2. Regression results of F18(2011, 2012) and F121999

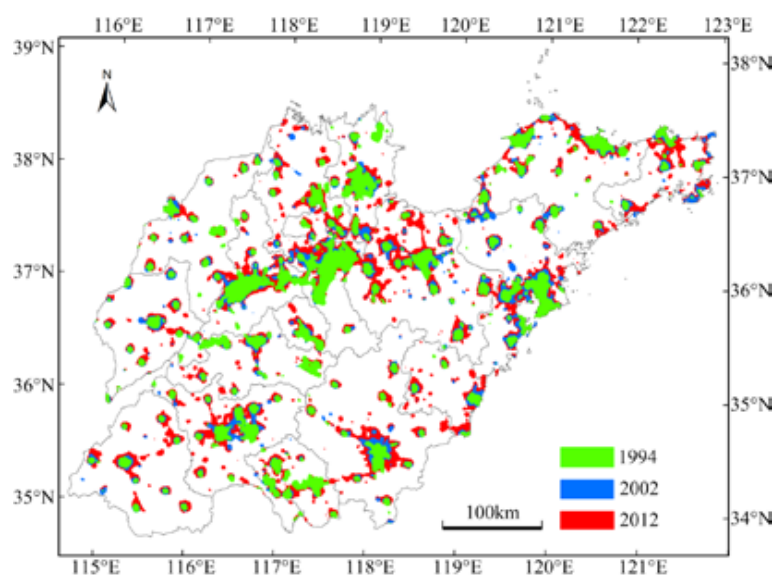

Figure 3. Urban changes of Shandong in 1994, 2002, and 2012 

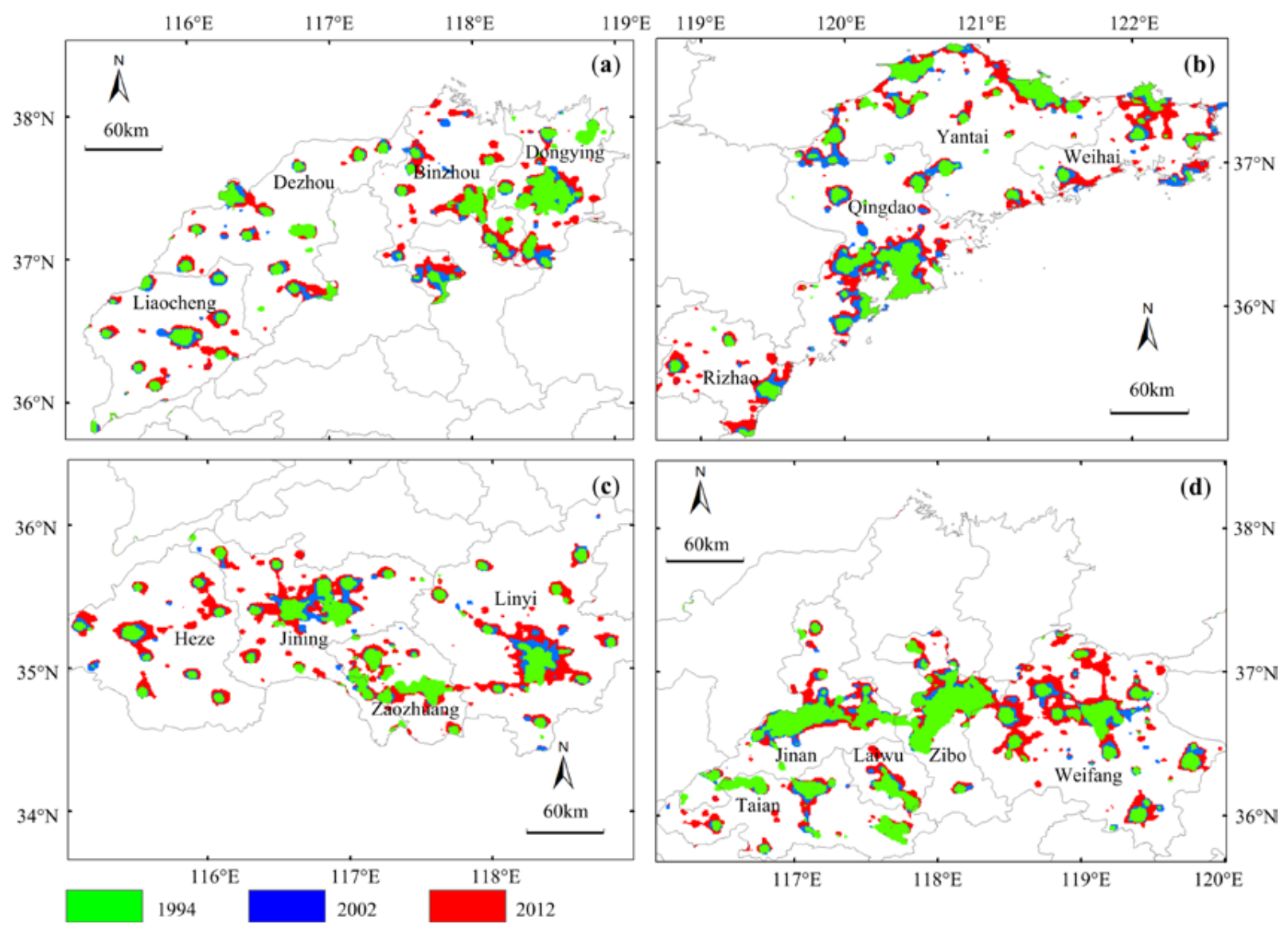

Figure 4. The urban areas of Shandong province in1994 (green), 2002 (blue), 2012(red): (a) the eastern coastal region; (b) the middle region; (c) the northern region; (d) the southern region

\subsection{Extractions}

There are various methods about extraction, such as empirical global threshold based methods and the mutation detection method. It is not easy to extract the data at a large spatial scale by the empirical threshold technique, because NSL data has the over-glow and over-saturated effects. The reliability and accuracy of ancillary data were key factors to ensure that this technique was successful (Henderson et al. 2003). Therefore, the value of the threshold is crucial to extract the urban information from NSL data. We used the threshold technique with ancillary to extract the data.

The subset within Shandong province area was clipped from the NSL data in each satellite year dataset. Urban areas and rural areas within administrative boundaries of each prefecture-level cities were extracted based on the clipping results. The threshold DN value was adopted when the minimum relative errors between the extracted area values and the statistical records were detected. The statistical data were provided by the Urban Statistical Yearbook of China (1993-2012). The value which was greater than the threshold represented the urban areas extracted by the experience threshold from NSL data. Comparisons of NSL data with resolution Landsat imagery on a wide variety of population density gradients indicated that the dimly lit areas with DN values less than 12 had agricultural and low population density land use (Small et al. 2011). The extraction results were presented in Figure 3 and Figure 4.

\subsection{Calculation}

FRAGSTATS is a computer software program designed to compute a wide variety of landscape metrics for categorical map patterns. It not only can compute numerous metrics that describe the structure of individual patches, classes or collections of patches of the same type, and the entire landscape mosaic for categorical patch mosaics, but also can compute a variety of cell-based metrics that describe the spatial structure of the local neighborhood of individual cells (McGarigal et al. 2012).

Based on the unicity of the landscape patterns which we extracted from the 17 prefecture-level cities in Shandong, we used FRAGSTATS4.2 to calculate nine landscape metrics, including the landscape total area (TA), the number of patches (NP), the largest patch index (LPI), the aggregation index (AI), the edge density (ED), the landscape shape index (LSI), the total edge length (TE), the mean patch area (AREA_MN) and the mean radius of gyration (GYRATE_MN).

\section{RESULTS AND DISCUSSION}

The results of the selected nine landscape metrics were shown in Figure 5. The number of patches (NP) reflects the fragmentation of the patches. Higher values of the number of patches correspond with higher level of the fragmentation of urban areas. The landscape shape index (LSI) indicates the divergence of the shape of a landscape patch from the circle which is considered ideal (Gyenizse et al. 2014). The 
aggregation index (AI) is used to measure aggregation levels of the landscape patches. The largest patch index (LPI) represents the ratio of the largest path to the landscape total area. The
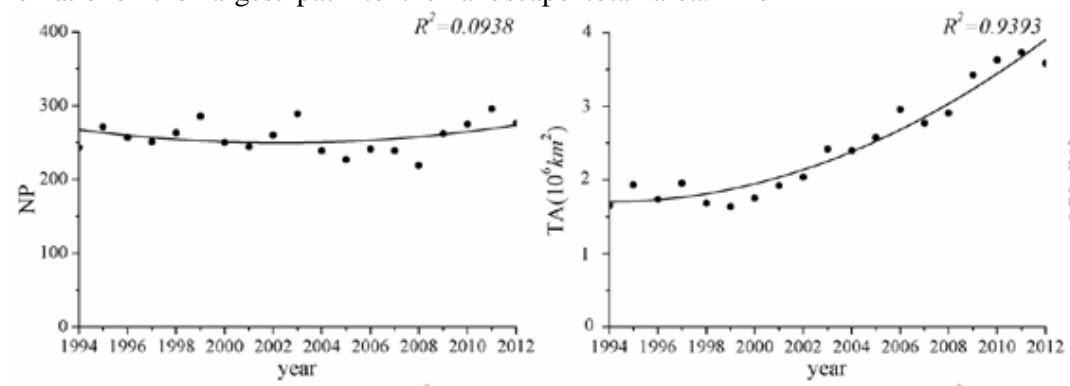

difference of urban areas which are in the same size.
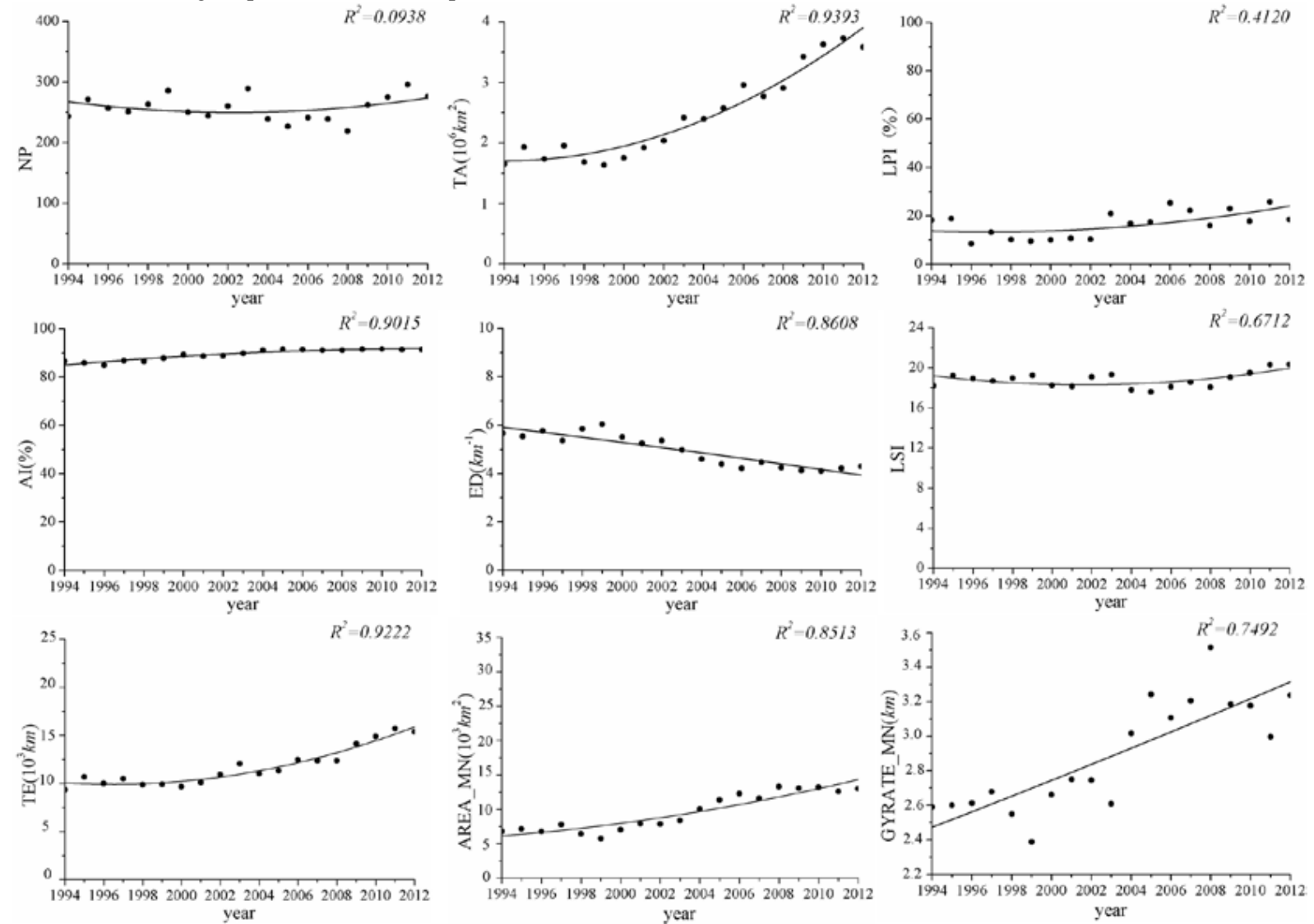

Figure 5. Trend chart of nine landscape metrics of Shandong from 1994 to 2012

\subsection{Results of Urban Expansion of Shandong Province}

The experimental results revealed that, from 1994 to 2012, the TA and the AERA_MN metrics of Shandong province increased roughly with certain unstable characteristics. The AERA_MN metric increased annually about $3.47 \%$ and the average annual growth rate of the GYRATE_MN metric was $1.18 \%$, which means Shandong province was in the process of urban expansion. The AI metric provides a quantitative basis to correlate spatial patterns of classes with specific processes. The value of AI metric was calculated among classes in the same landscape allow reasonable comparisons as an index to quantify the level of aggregation of spatial patterns (He el at. 2000). The AI metric of Shandong province suggested the neighboring cities tended to integrate a whole. The average area of urban patches in this area was increased by $4.17 \%$ per year. In the last 19 years, the NP metric decreased marginally from 1999 to 2000 while it tended to increase after 2000. The NP metric reached the peaks at 1998, 2003 and 2011, which suggested that the boom towns emerged periodically and merged into an urban area after several years. The reason behind this phenomenon was the implementation of the policies of urban construction in the 90 's and speeding up the urbanization in the $21^{\text {st }}$ century (Figure 5).

As suggested above, from 1994 to 2012, Shandong was in the development of urbanization with the emergence of the boom towns. As for the primary factors of urban areas' increase, the expansion of the cities and the merger of neighboring cities made great contributions.

The LPI metric tended to increase from 1994 to 2012, which could deduced that, as one of the largest cities in Shandong province, the urban area of Jinan was increasingly expanding. This demonstrated that the expansion of the large cities played a dominated role in urban expansion in this region. At the same time, the average annual growth rate of the TE metric was $2.67 \%$ while the ED metric decreased by $1.44 \%$ annually, which verified that the merger of neighboring cities was one of the major factors to influence the urbanization of Shandong province.

\subsection{Result of Typical Prefecture-level Cities}

In view of the 17 prefecture-level cities' similarities in urbanization, we selected eight typical cities in different regions of Shandong province.

As an energy-supplying city, Dongying was rich in oil reserves. From 1994 to 2012, the total area of urbanized area in Dongying kept increasing. The number of patches metric had a regular change, which meant the boom towns integrated each other every several years. But the largest patch index metric didn't have large changes and fluctuations with the rate between $58.34 \%$ and $76.32 \%$; it indicated that the expansion of developed regions was one explicit reason of the urban area expansion. The largest patch index metric decreased every year, and combined with the mean patch area metric in Figure 6, it 
revealed that the emergences and expansions of the boom towns were other important factors for urban expansion.

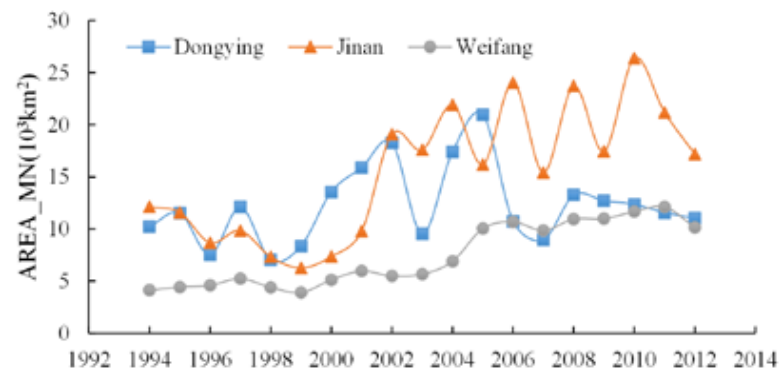

Figure 6. The variation of AREA_MN metric of Dongying, Jinan, and Weifang

Unlike Dongying, Liaocheng is one of those cities which rely on the development of agriculture on western plain in Shandong. The annual increment velocity of the landscape total area metric of Dongying was 1.78 times faster than Liaocheng, which indicated that modern industries performed more significant effects on promoting the urbanization procedures. As shown in Figure 7, the largest patch index metric met an obvious drop from $34.40 \%$ to $23.27 \%$ in the period of $2001-2009$. The mean patch area metric revealed that boom towns in Liaocheng area expanded at a relatively stable speed every year. But the rate of the largest patch index metric went up from $22.76 \%$ to $37.30 \%$ during 2010 to 2012, which reflected a large scale of integrations of boom towns (Figure 7). From 1994 to 2012, the main reasons of Liaocheng's urbanization were emergences and expansion of the boom towns.

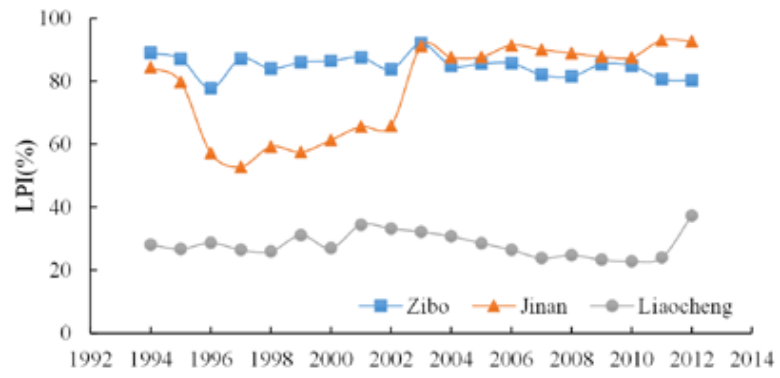

Figure 7. The variation of LPI metric of Zibo, Jinan, and Liaocheng

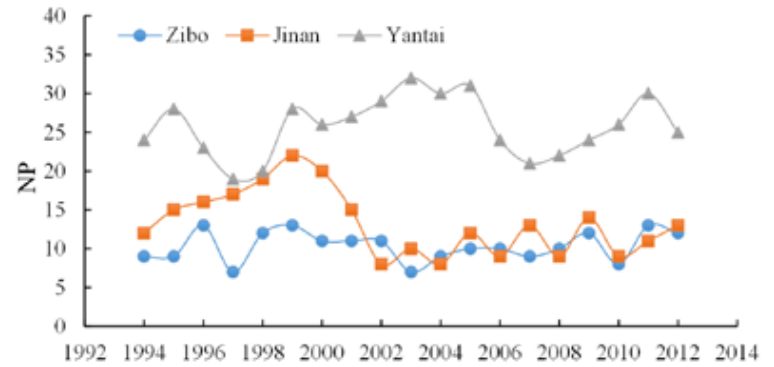

Figure 8. The variation of NP metric of Zibo, Jinan, and Yantai

Jinan is the capital of Shandong province. As shown in Figure 7 and Figure 8, from 1994 to 2002, the LPI metric and the NP metric reflected that the number of the boom towns was continuously increased annually, as well as the total urban area values. This phenomenon resulted in a decline of the largest patch index metric. With the newly boom towns dissolving into a largest patch, the LPI metric soared from $65.81 \%$ to $91.14 \%$ in 2003 (Figure 7). From 2002 to 2012, the rising TA metric revealed that the urban area was persistently expanding. Meanwhile, the NP metric and the AREA_MN metric had a periodic change which meant the perpetual emersions of the boom towns and their merging with developed areas. The largest patch index metric was still at $92.61 \%$, which indicated that the expansion of the developed urban area of Jinan city was the main reason for the urban expansion during 2002-2012.

As a regional core city in East China, Qingdao lies on eastern coastal region of Shandong province. The LPI metric and the NP metric revealed that Qingdao was at such a state which contained emergences, expansion and integration of the boom towns during 1994 to 2012. Until 2009, Qingdao had a relatively stable largest urban patch with the rate of $79.50 \%$. The number of patches and the mean patch area metric reflected that the expansion speeds of the boom towns were relatively more uniform than Jinan. From 1994 to 2012, emergences and expansion of the boom towns were the major forces to push Qingdao’s urbanization.

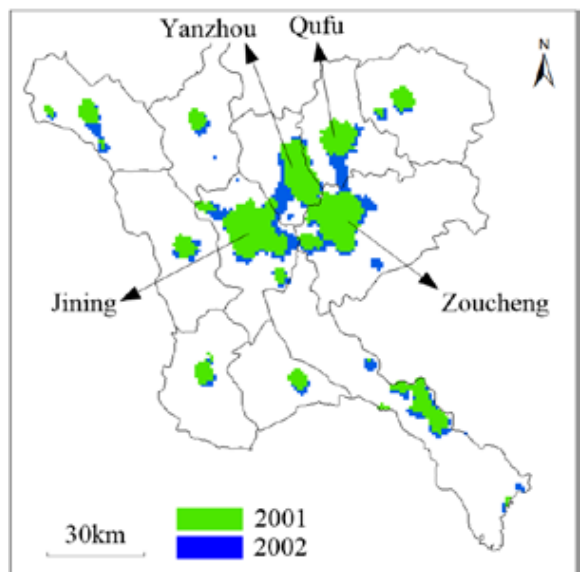

Figure 9. The integration phenomenon of county-level cities in Jining from 2001 to 2002

Jining and Yantai are both famous tourist cities. The former is rich in coal resources and the latter is famous for its advanced levels of the secondary industries and classic agricultural products in Shandong province. From 1994 to 2009, for Jining, the NP metric's value was high with the average of 14.6, and the variation of the LPI metric presented that the number of the boom towns increased annually. With the expansion and integration of the boom towns, Jining had a relatively stable largest patch area in 2002 with the rate of $66.27 \%$. However, the LPI metric was decreased because of the expansion of the boom towns. As shown in Figure 9, the merging of county level cities with the developed area of Jining city caused extraordinary raise to the metric of LPI.

From 1994 to 2012, the landscape total area of Yantai in Figure 10 was always larger than about 1 time the landscape total area of Jining. But the value of the NP metric was higher than Jining with the average number of 27.6 in Figure 8. The gentle variation of the LPI metric and the increase trends of the AREA_MN metric of Yantai reflected that the expansion velocities of the boom towns were relatively slow. The mean patch area metric grew by $45.72 \%$ from 2005 to 2006 which revealed that the expansion of the developed urban area was the main reason for Yantai's urbanization. The expansion and integration of the boom towns were at a relatively sluggish pace. Until 2012, Yantai had a relatively stable largest patch with the rate of $57.64 \%$. 
Zibo is one of traditional industrial cities in the middle region of Shandong province. As shown in Figure 8 and Figure 7, the NP metric and the LPI metric indicated that, from 1994 to 2012, the rate of the largest patch was accounted a proportion of approximately $80 \%$. The landscape total area metric in Figure 10 and the mean patch area metric reflected that, the expansion of existing urban area was the major factor for the urbanization procedure, although the boom towns continued to increase and expand. The growth rate of the landscape total area metric dropped to $0.58 \%, 0.22 \%$, and $-2.22 \%$ from 2010 to 2012, which revealed that the urban area of Zibo city expanded tardily in this period (Figure 10).

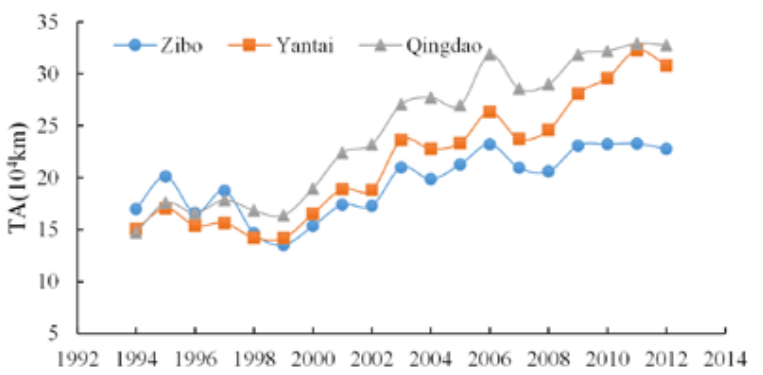

Figure 10. The variation of TA metric of Zibo, Yantai and Qingdao

The number of patches metric and the largest patch index metric of Weifang city revealed that, from 1994 to 2006, the emergency and expansion of the boom towns in this region made a rapid increase of urban areas. Besides, the gentle increase variation pattern of Weifang's AREA_MN metric revealed that the speeds of boom towns' expansions were relatively uniform in Figure 6. Until 2006, Weifang had a relatively stable largest patch with the rate of $63.73 \%$, but it was still influenced by the expansion of boom towns with large areas.

\section{CONCLUSIONS}

In this study, we used the DMSP/OLS nighttime luminosity data to extract the dynamics of urban expansions in Shandong province. The experimental results indicated that, from 1994 to 2012, with the development of urbanization in Shandong, the total urban area of cities expanded for 1.17 times, the average urban area of cities in this region increased by about $93.00 \%$, the average annual growth rate of total edge lengths is $2.67 \%$, while the edge density decreased about $1.44 \%$ annually, which means the neighboring cities tended to integrate a whole. The energy-supplying cities in Shandong province revealed relative higher urbanization velocities than the cities rely on the development of the primary industries, like Liaocheng, Heze, and Dezhou. Traditional industrial cities, such as Zibo and Jinan, presented slower speeds in the urbanization development processes than resort cities, such as Yantai and Jining. Regional boom cities like Weifang have accelerated the process of urbanization expansion since 2000. Coastal metropolis of Qingdao city represented much more rapid expansion velocities than those inland bigger cities, including Jinan, Weifang, and Jining. In the middle area of Shandong province, the connectivity between developed urban areas was constantly increased. The phenomenon was closely related to the emergences of the boom towns, the expansion of developed urban areas, and the integration of neighboring towns. As suggested above, Shandong was in the development of rapid urban expansion.

The DMSP/OLS luminosity data provided a rapid, efficient, and easy-to-acquire data source for mapping urban areas in multiple spatial scales. However, our experimental results also revealed that the data intercalibration method adopted in this report might not completely solve the data comparability problem among inter-annual nighttime imageries. The design and implementation of advanced calibration methods and solutions may be an essential aspect to improve the analysis results of the nighttime stable light data.

\section{ACKNOWLEDGEMENTS}

This research has been supported by the National Natural Science Foundation of China (Grant No. 41501425), the Key Research and Development Program of Shandong Province (2016GSF122006; 2015GSF122008), the Project of Shandong Province Higher Educational Science and Technology Program (J16LH03), the Young Teacher Development Support Program of Shandong University of Technology (4072-115016), and the State Key Laboratory of Resources and Environmental Information System.

\section{REFERENCES}

Amaral, S., Câmara, G., Monteiro, A. M., Quintanilha, J. A., and Elvidge, C. D., 2005. Estimating population and energy consumption in Brazilian Amazonia using DMSP night-time satellite data. Computers, Environment and Urban Systems, 29(2), pp. 179-195.

Deng Y., Liu S., Cai J., et al., 2015. Spatial pattern and its evolution of Chinese provincial population: Methods and empirical study. Journal of Geographical Sciences, 25(12), pp. 1507-1520.

Doll, C.N.H., Muller, J.-P., Morley, J.G., 2006. Mapping regional economic activity from night-time light satellite imagery. Ecological Economics, 57(1), pp. 75-92.

Elvidge, C.D., Baugh, K.E., Kihn, E.A., et al., 1997. Mapping city lights with nighttime data from the DMSP operational linescan system. Photogrammetric Engineering \& Remote Sensing, 63(6), pp. 727-734.

Elvidge C. D., Ziskin D., Baugh K. E., et al., 2009. A fifteen year record of global natural gas flaring derived from satellite data. Energies, 2(3), pp. 595-622.

Elvidge C D, Baugh K E, Dietz J B, et al., 1999. Radiance calibration of DMSP-OLS low-light imaging data of human settlements. Remote Sensing of Environment, 68(1), pp. 77-88.

Elvidge, C. D., Baugh, K. E., Kihn, E. A., Kroehl, H. W., Davis, E. R., and Davis, C. W., 1997. Relation between satellites observed visible-near infrared emissions, population, economic activity and electric power consumption. International Journal of Remote Sensing, 18(6), pp. 1373-1379.

Fan, J., Ma, T., Zhou, C., et al., 2014. Comparative estimation of urban development in China's cities using socioeconomic and DMSP/OLS night light data. Remote Sensing, 6(8), pp. 7840-7856. 
Gyenizse P, Bognár Z, Czigány S, et al., 2014 Landscape shape index, as a potential indicator of urban development in Hungary. Landscape \& Environment, 8(2), pp.78-88

Henderson, M., Yeh, E. T., Gong, P., Elvidge, C., \& Baugh, K. E., 2003. Validation of urban boundaries derived from global night-time satellite imagery. International Journal of Remote Sensing, 24(3), pp. 595-609.

He, H. S., Dezonia, B. E., \& Mladenoff, D. J., 2000. An aggregation index (AI) to quantify spatial patterns of landscapes. Landscape Ecology, 15(7), 591-601.

Liu, Z., He, C., Zhang, Q., Huang, Q., \& Yang, Y., 2012. Extracting the dynamics of urban expansion in China using DMSP-OLS nighttime light data from 1992 to 2008. Landscape and Urban Planning, 106(1), pp. 62-72.

Ma A., Wang J., and Zhang K. 2012a. Chapter 2: Sampling Survey of Heavy Metal in Soil Using SSSI. Yeh A., et al. (eds.), Advances in Spatial Data Handling and GIS, Lecture Notes in Geoinformation and Cartography, DOI:10.1007/978-3-64225926-5_2, Springer-Verlag, Berlin Heidelberg, pp. 15-26.

Ma T., Zhou C., Pei T., et al., 2012b. Quantitative estimation of urbanization dynamics using time series of DMSP/OLS nighttime light data: A comparative case study from China's cities. Remote Sensing of Environment, 124, pp. 99-107.

Ma T., Yin Z., Li B., et al., 2016. Quantitative estimation of the velocity of urbanization in China using nighttime luminosity data. Remote Sensing, 8(2), 94. doi:10.3390/rs8020094.

McGarigal K., SA Cushman, and E Ene. 2012. FRAGSTATS v4: Spatial Pattern Analysis Program for Categorical and Continuous Maps. Computer software program produced by the authors at the University of Massachusetts, Amherst. Available at the following web site: http://www.umass.edu/landeco/resear ch/fragstats/fragstats.html (15 April 2016)

National Bureau of Statistics of China. 2016, National Data. http://data.stats.gov.cn/easyquery.htm?cn=E0103 (7 May 2016)

Peng, J., Liu, Z., and Liu, Y., Wu, J., and Han, Y., 2012. Trend analysis of vegetation dynamics in Qinghai-Tibet Plateau using Hurst Exponent. Ecological Indicators., 14(1), pp. 28-39.

Sutton, P.C., 2003. A scale-adjusted measure of "urban sprawl" using nighttime satellite imagery. Remote Sensing of Environment, 86(3), pp. 353-369.

Small, C., Elvidge, C. D., Balk, D., \& Montgomery, M. R. 2011. Spatial scaling of stable night lights. Remote Sensing of Environment, 115(2), pp. 269-280.

Small, C., Pozzi, F., Elvidge, C.D., 2005. Spatial analysis of global urban extent from DMSP-OLS nightlights. Remote Sensing of Environment, 96(3-4), pp. 277-291.

Wikipedia. 2016, Shandong. https://en.wikipedia.org/wiki/Shan dong (4 March 2016).

Xiong, Y., Huang, S., Chen, F., et al., 2012. The Impacts of Rapid Urbanization on the Thermal Environment: A Remote Sensing Study of Guangzhou, South China. Remote Sensing, 4(7), pp. 2033-2056.
Xu, T., Ma, T., Zhou, C., et al., 2014. Characterizing spatiotemporal dynamics of urbanization in China using time series of DMSP/OLS night light data. Remote Sensing, 6(8), pp. 77087731.

Yang R., Liu Y., Long H., et al., 2015. Spatio-temporal characteristics of rural settlements and land use in the Bohai Rim of China. Journal of Geographical Sciences, 25(5), pp. 559-572.

Zhang, Q., Seto, K.C., 2011. Mapping urbanization dynamics at regional and global scales using multi-temporal DMSP/OLS nighttime light data. Remote Sensing of Environment, 115(9), pp. 2320-2329.

Zhou, Y., Ma, T., Zhou, C. et al., 2015. Nighttime light derived assessment of regional inequality of socioeconomic development in China. Remote Sensing, 7(2), pp. 1242-1262.

Zhou, Y., Smith, S.J., Elvidge C.D., et al., 2014. A clusterbased method to map urban area from DMSP/OLS nightlights. Remote Sensing of Environment, 147(18), pp. 173-185. 